\title{
FACTORES INFLUYENTES EN EL DISEÑO DE UNA CAMPAÑA DE MARKETING DIGITAL PARA UNA MARCA DE MAQUILLAJE
}

\author{
INFLUENCING FACTORS IN THE DESIGN OF A DIGITAL \\ MARKETING CAMPAIGN FOR A MAKEUP BRAND
}

\section{FATORES INFLUENCIADORES NO DESENHO DE UMA CAMPANHA DE MARKETING DIGITAL PARA UMA MARCA DE MAQUIAGEM}

\author{
Gustavo Adolfo Rubio Rodríguez ${ }^{1}$ \\ Fernando de Almeida Santos ${ }^{2}$ \\ Edwin Giovanny Ruiz Muriel ${ }^{3}$ \\ Juan José Cabrera Guzmán ${ }^{4}$ \\ Artigo recebido em janeiro de 2021 \\ Artigo aceito em agosto de 2021
}

\begin{abstract}
RESUMEN
El objetivo de la presente investigación consiste en determinar los factores que inciden en el diseño de una campaña de marketing digital para una marca de maquillaje. Como instrumento de recolección de información se empleó la encuesta, la cual fue administrada a 460 personas de una población estimada de 20.000 usuarios de los canales e-commerce (redes sociales, suscriptores web). Los hallazgos encontrados corresponden a que los hábitos de compra, horarios, el comportamiento de los usuarios motivados por las tendencias de las redes sociales, el tipo de contenido digital, la preferencia de canales de pago como botón PSE, los links de pasarelas de pago y en efectivo, entre otros, son fundamentales para perfilar una estrategia enfocada a los consumidores de campañas digitales de maquillaje. Como conclusión, es fundamental conocer estos factores para la identificación del consumidor y a su vez evaluar el diseño y lanzamiento de campañas; de igual forma, es imprescindible segmentar adecuadamente el mercado, conocer las tendencias, preferencias de los usuarios, influencers que los motivan, para finalmente diseñar de manera exitosa la campaña propuesta.
\end{abstract}

Palabras claves: Redes sociales. Marketing digital. E-commerce. Maquillaje.

\section{ABSTRACT}

The objective of this research is to determine the factors that influence the design of a digital marketing campaign for a makeup brand. The survey was used as an instrument for collecting information, which was administered to 460 people out of an estimated population of 20,000 users of e-commerce channels (social networks, web subscribers). The findings found correspond to the fact that shopping habits, schedules, user behavior motivated by social media trends, type of digital content, preference of payment channels such as PSE button, links to payment gateways and Cash, among others, are essential to outline a strategy focused on consumers of digital makeup campaigns.

\footnotetext{
${ }^{1}$ Corporación Universitaria Minuto de Dios, Colômbia. E-mail: gustavo.rubio-r@uniminuto.edu.co.

${ }^{2}$ Pontifíícia Universidade Católica de São Paulo. E-mail: fernando@ @ernandoasantos.com.br.

${ }^{3}$ Universidad del Tolima, Colômbia. E-mail: egruizm@ut.edu.co.

${ }^{4}$ Universidad del Tolima, Colômbia. E-mail: jjcabrera@ut.edu.co.
} 
In conclusion, it is essential to know these factors for the identification of the consumer and in turn evaluate the design and launch of campaigns; in the same way, it is essential to properly segment the market, know the trends, user uinfluencers that motivate them, to finally design the proposed campaign successfully.

Keywords: Social media. Digital marketing. E-commerce. Makeup.

\section{RESUMO}

O objetivo desta pesquisa é identificar quais s fatores que influenciam o desenho de uma campanha de marketing digital para uma marca de maquiagem. A pesquisa coleta informações de 460 respondentes, de uma população estimada de 20 mil usuários de canais de comércio eletrônico (redes sociais, assinantes da web). Os resultados encontrados demonstram que os hábitos de compra, os horários e o comportamento do usuário são motivados pelas tendências das mídias sociais, pelo tipo de conteúdo digital, pela preferência dos canais de pagamento como o botão PSE, sendo que os links dos gateways de pagamento e Dinheiro, entre outros, são essenciais para traçar uma estratégia voltada para as consumidoras de campanhas de maquiagem digital. Em conclusão, é fundamental conhecer estes fatores para a identificação do consumidor e, por sua vez, avaliar o desenho e lançamento das campanhas. Da mesma forma, é fundamental segmentar adequadamente o mercado, conhecer as tendências, as preferências dos usuários, os influenciadores que os motivam, para finalmente desenhar a campanha proposta com sucesso.

Palavras-chave: Redes sociais. Marketing digital. E-commerce. Maquiagem.

\section{INTRODUCCIÓN}

En el último siglo las tendencias de compra han pasado por diferentes etapas; la mentalidad ha trascendido desde un enfoque de la productividad limitada a un contexto social y geográfico a un enfoque más global. En la actualidad se han generado fenómenos sociales y tecnológicos que, aunados a la aparición de plataformas tecnológicas, han llevado a las redes sociales a una maduración (Sainz, 2018). Asimismo, Sanmiguel (2020), afirma que cada vez es más necesario conectar con los consumidores, escucharlos, dialogar con ellos y conocer sus verdaderos intereses, necesidades e inquietudes; el conocimiento de los clientes es fundamental en el marketing digital.

En consecuencia, en Colombia, la cultura de compra digital es relativamente nueva comparada con países como Brasil, México y Argentina, líderes en transacciones digitales en Centro y Sur América. Colombia se encuentra en el quinto lugar después de Chile; cada año se encuentran procesos de compra más simples en plataformas digitales. Por lo tanto, los colombianos prefieren el comercio electrónico debido a que encuentran un mayor y más accesible portafolio de productos y servicios; en los últimos cinco años el E-commerce ha tenido un crecimiento exponencial, se estima que en 2021 se alcanzarán ventas superiores a USD \$26.073 millones en Colombia (Statista, 2020).

En este sentido, la emergencia sanitaria del COVID-19, ha transformado la vida de millones de personas en el mundo; el aislamiento preventivo obligatorio ha llevado a que las personas deban permanecer en sus hogares. Las plataformas de comercio electrónico han contribuido a mantener el abastecimiento, facilitando el cumplimiento de las medidas de distanciamiento social (Cámara Colombiana de Comercio Electrónico, 2020). Bajo esta perspectiva, un comercio electrónico requiere de conocimientos estratégicos a la hora de ejecutar campañas de marketing digital. El E-commerce, E-business, E-marketing, consolidan lo que llamamos el marketing digital o marketing viral. 
En efecto, las plataformas digitales permiten segmentar de una manera eficiente el mercado, ya que los sistemas digitales facilitan crear perfiles detallados de los usuarios de internet, con el fin de conocer sus gustos y preferencias (Selman, 2017). Del mismo modo, Torres (2020), resalta la importancia de saber usar las herramientas tecnológicas para propiciar nuevas formas de trabajo más agiles, interactivas y colaborativas. Con el auge de la tecnología se aprecia que varias pequeñas y medianas empresas se aventuran en el Ecommerce, desconociendo la dinámica de este tipo de comercio (Méndez, 2013).

Por lo anterior, el marketing digital ha cambiado el modo de hacer que las empresas lleguen con sus productos y servicios a los consumidores, afirmando que la utilización de herramientas digitales es de gran ayuda para llegar incluso a nichos de mercado más específicos con menores costos y en tiempo real (Valencia et al. 2014). Asimismo, se resalta que no solo se trata de cómo manejar las herramientas, sino, de entender un nuevo mundo digital en el cual el eje central es el consumidor (Selman, 2017; Moschini, 2012).

Igualmente, una campaña de marketing digital enfocada en redes sociales, genera un impacto positivo en términos de ingresos por ventas, reconocimiento y posicionamiento de marca. Del mismo modo, se desea crear una comunidad de marca en la cual los consumidores puedan compartir experiencias, trabajar en red con personas afines e involucrarlos en el desarrollo de las ideas y productos de la compañía para finalmente llegar al marketing $\mathrm{H} 2 \mathrm{H}$ (persona a persona), (Fonseca, 2014).

Finalmente, se tiene como objetivo determinar los factores que inciden en el diseño de una campaña de marketing digital para una marca de maquillaje. Estos factores permitirán identificar el perfil del consumidor para incorporarlo en la estrategia de marketing digital, para posteriormente ser desarrollada e implementada en las campañas de redes sociales y canales E-commerce. Esta investigación cobra vital importancia, dado que la información recolectada consentirá la construcción de metodologías, estrategias y pautas para caracterizar integralmente el cliente de maquillaje.

\section{MARCO TEÓRICO}

Comienza el marco teórico del marketing digital.

\subsection{Marketing digital}

Son un conjunto de estrategias de mercadeo, totalmente digitales y que pretenden orientar al usuario a transformarse digitalmente, integra conceptos de comunicación, publicidad, relaciones públicas, computación y lenguaje, además se caracteriza por la personalización y la masividad del contenido (Selman, 2017). Por lo tanto, el marketing digital agrupa una serie de conceptos adicionales que son capaces de personalizar la oferta de acuerdo a las necesidades del consumidor interactuando con los indicadores de crecimiento y estudiando cada vez más al cliente. Uno de los canales predeterminados por parte de las plataformas de marketing son las aplicaciones de mensajería y chat box, que permiten una respuesta inmediata a los requerimientos de los clientes, generando contenido atractivo, único y diferenciado (Andrade, 2016).

De acuerdo con Mora et al. (2019), para las pymes el desafió tecnológico que representa implementar el marketing digital y conocer su importancia debe ir más allá de 
impulsar las ventas. Por tanto, se deben de implementar procesos de innovación que contribuyan en el crecimiento de las estrategias digitales y en la eliminación de barreras económicas y operativas en la aplicación de herramientas TIC y el plan de marketing. Las pymes en Colombia proporcionan el $28 \%$ del PIB, el $67 \%$ del empleo y el $37 \%$ de la producción nacional; en este sentido, representan gran capacidad de oferta productiva del país, siendo las llamadas a generar desarrollo y progreso en las regiones. Sin embargo, solo el $7,4 \%$ realiza comercio electrónico, por lo que la digitalización es una de las prioridades de crecimiento empresarial siendo un factor determinante en los planes de desarrollo gubernamentales (Shirley \& Sastoque, 2020).

Las herramientas que ofrece el marketing digital para posicionarse de manera online son diversas y accesibles para desarrollar la presencia en los medios digitales. Entre ellas se encuentra wordpress, wix, jimdo, jsuite, vook, etc., que permiten iniciar el camino del marketing digital sin tener que realizar grandes inversiones, generando indicadores que perfilen analíticas y dirijan nuevas estrategias mediante promoción y publicidad de acuerdo con el target identificado (Ortega, 2020). Asimismo, el marketing digital ha cambiado y mejorado el entorno de los negocios, migrando desde el comercio electrónico al comercio digital convirtiéndose en una herramienta de apoyo a la competitividad mundial.

Del mismo modo, el marketing digital ha facilitado la comercialización de bienes y servicios mediante plataformas de pago electrónico, cambiando el dinero tradicional por la banca virtual, de tal modo, que se logró un incremento de los niveles de confianza y culturización en pagos por botón PSE, Paypal, PayU, Wompy, entre otros (Perdigón et al. 2018). Para tener presencia on line se pueden abarcar diferentes plataformas sociales, con el objetivo de alcanzar mayor cantidad de usuarios, pero entre más herramientas se elijan en la estrategia digital, mayor exigencia y control se requiere para administrar el contenido y lograr atender oportunamente los clientes. Para esto se sugiere integrar todas las redes sociales con herramientas como: social media Poster SEMrush, Hootsuite, CoSchedule, las cuales permiten administrar todo el contenido de las redes desde una sola plataforma, incluso estableciendo horarios y agendando publicaciones en las horas pico de conexión del público objetivo (Desai \& Shah, 2019).

Un aspecto importante para la planeación del marketing digital es entender el valor agregado de la organización; de hecho, los consumidores no adquieren productos sino soluciones. Si estas superan las expectativas se produce una migración de consumidor a cliente, dónde pesa más el posicionamiento de marca, que otros factores del mercado como precio y calidad. Adicionalmente, los negocios más allá de vender productos se comprometen con generar bienestar basados en la identificación de necesidades, campañas de expectativa, ofertas ganadoras y promesas dónde se cautiva la audiencia mediante lenguaje, material audiovisual y experiencia emocional (Deiss \& Henneberry, 2020).

De manera análoga, el marketing relacional desempeña un papel fundamental y contribuye a la consolidación en la relación con los clientes, iniciando desde etapas de apropiación del nicho y tácticas que consoliden y vinculen grupos de interés, empleados y competidores. Del mismo modo, establecer relaciones interpersonales permite desarrollar un lenguaje realista que propenda por obtener una relación de largo alcance, con beneficios para todas las partes. En consideración con lo anterior los retornos de la inversión para las compañías se vuelven más rentables y la relación con el cliente incrementa unidimensionalmente, siendo directamente proporcional y rentable para ambas partes (Rasmussen, 2018).

Igualmente, la conversión digital en el relacionamiento de marketing no distingue de edades, preferencias o estratos sociales. Plataformas de Facebook y Google están abiertas al 
público para migrar hacia lo digital (Dodson, 2016). De acuerdo con Vargas (2017), el marketing digital se posiciona como el nuevo rumbo de desarrollo que simplifica procesos, ahorra tiempo y agiliza respuestas a necesidades puntuales, materializando oportunidades y generando procesos de inversión por más de USD 660 millones en Colombia. Ante la situación actual de la pandemia por Covid19, este tipo de forma de mercadeo se convierte en una oportunidad latente y necesaria para las empresas.

Dado lo anterior, la próxima etapa del marketing digital es consolidar estrategias de remarketing, incluyendo ofertas especiales, contenido adicional, cupones y otras iniciativas que vuelvan a cautivar a los consumidores sin demostrar que le apuntan a una transformación comercial. Esto puede incrementar la fidelización de los consumidores y ampliar la comunicación entre los clientes y las empresas, generando beneficios y ganancias en términos de relaciones de largo plazo (López et al. 2019). Finalmente, seis grandes retos en términos de sostenibilidad representan que las plataformas cuenten con procesos de investigación para aplicar procesos de mejora, enfocándose en la atracción de nuevos clientes y las tendencias emergentes: orientación de clientes y propuesta de valor, mentalidad digital, marketing verde, ventaja competitiva, cadena de suministro y capacidades (Diez et al. 2019).

\section{MÉTODO}

Esta investigación empleó un método cuantitativo mediante la realización de un muestreo probabilístico aleatorio simple. Según Otzen \& Manterola (2017) "todos los individuos que componen la población tienen la misma oportunidad de ser incluidos en la muestra. La probabilidad de selección de un sujeto es independiente de la probabilidad que tienen el resto de los sujetos que integran la población" (p.35). Por tanto, se seleccionaron sujetos al azar de una población de interés para el estudio propuesto.

\subsection{Población}

La población objeto de estudio corresponde a 20.000 personas del territorio colombiano, entre 16 y 60 años, usuarios de las redes sociales de una marca de maquillaje, con poder de decisión de compra de este tipo de artículos. Los datos para procesar en la investigación son de carácter privado y serán insumo importante para la generación de estrategias de ejecución por parte del área de mercadeo de la compañía estudiada y/o de las marcas de la misma industria o segmentos afines. De igual forma, algunos resultados pueden extrapolarse para ser usados en sectores similares al estudiado y en otros contextos geográficos.

\subsection{Marco muestral}

El marco muestral de la presente investigación es una lista de los usuarios de las redes sociales (Instagram, Facebook, WhatsApp) y suscriptores de la página web de una empresa de maquillaje en Colombia. Por razones de confidencialidad de la información, esta lista no puede ser publicada en el contenido de este manuscrito; pero, para efectos de su veracidad puede ser constatada confirmando el número de seguidores de las redes sociales de la marca Mirely makeup. 


\subsection{Muestra}

La muestra la constituyeron 460 personas a nivel nacional, pertenecientes a las redes sociales de una empresa del sector de maquillaje, cosméticos y artístico, derivada del procedimiento matemático presentado en la Figura 1, en el que se estima un margen de error del $5 \%$ y un nivel de confianza del 97\%. Los resultados obtenidos de esta muestra contribuirán a identificar los factores influyentes en las campañas de marketing, los cuales a su vez serán útiles para fortalecer las estrategias de un plan de marketing digital.

Figura 1 - Cálculo de la muestra

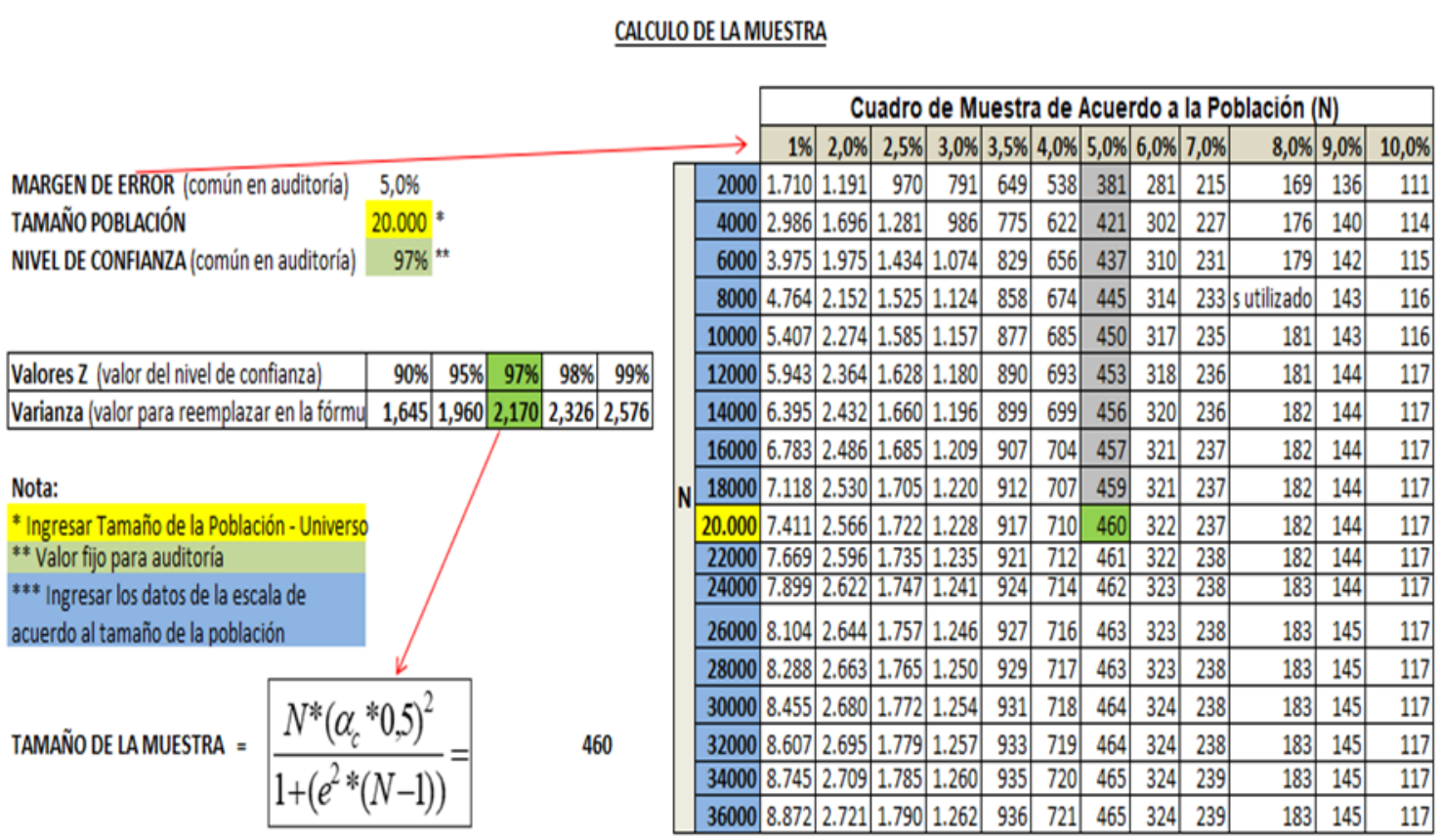

Elaborado por los autores

Donde:

$\alpha_{c}=$ Valor del nivel de confianza (varianza)

Nivel de confianza: es el riesgo que se acepta de equivocarse al presentar los resultados (también se puede denominar grado o nivel de seguridad). El nivel habitual de confianza es del $95 \%$, para este caso fue del $97 \%$.

$e=$ Margen de error

Margen de error: es el error que se está dispuesto a aceptar al equivocarse al seleccionar la muestra; este margen de error para este caso fue del $5 \%$.

$N=$ Tamaño de la Población (universo)

\subsection{Instrumento}

Un instrumento en metodología de investigación es una herramienta que vincula los actores con el objeto de la investigación; es fundamental, ya que este permite conectar la lógica con el conocimiento técnico de los aspectos a analizar (Hernández, Fernández, \& 
Baptista, 2014). La técnica empleada para la recolección de información fue la encuesta, la cual fue administrada de forma digital aleatoriamente a 460 participantes, con edades comprendidas entre los 16 y 60 años, localizados en toda Colombia.

La encuesta contiene preguntas estratégicamente planteadas, cuya respuesta permite identificar factores influyentes y decisivos en la realización de campañas de marketing digital para el sector del maquillaje, lo cual, a su vez, motivan o impactan en la intención de compra de los usuarios de redes sociales. Algunos de los factores analizados fueron los horarios frecuentes de compra, motivación del consumidor, tendencias, comportamiento de compra, psicología del consumidor, frecuencia de compra, preferencias en los servicios de venta y postventa, tipo de herramientas de pago preferidas, etc.

El cuestionario se administró a través varios mecanismos: Redes sociales (Instagram, Facebook, WhatsApp), mediante la herramienta de en vivos y banners que contenían el link de acceso al formulario, el cual una vez es diligenciado de manera aleatoria por los participantes, cada respuesta es guardada automáticamente. También se enviaron banners mediante la herramienta de email marketing (el sistema envía los correos aleatoriamente) a los suscriptores de la página principal de maquillaje, los cuales también contenían el link de acceso a la encuesta. Una vez completada la muestra que arrojo el cálculo estadístico se procedió a cerrar la encuesta en línea.

\subsection{Tratamiento de la información}

A partir de los resultados obtenidos de la encuesta, se procedió a tabular la información mediante el programa SPSS, generando representaciones graficas que fueron interpretadas y luego analizadas. Con el fin de soportar estos resultados y brindar mayor veracidad a esta información, se realizó una extensa revisión bibliográfica la cual se contrastó y valido con investigaciones y teoría relacionada con el tema.

Adicionalmente, se determinó la moda en cuanto a la palabra clave más usada para buscar los artículos de maquillaje, y además se identificaron las cinco palabras determinantes más utilizadas por los encuestados, con el fin de proponerlas como palabras estratégicas de las herramientas de SEO y SEM marketing.

Con esta información se pretende, sirva de herramienta para el diseño de campañas de marketing digital orientadas al comercio electrónico, o bien las empresas interesadas puedan aplicar una herramienta similar; es decir, sigan la metodología empleada en la presente investigación para obtener información relevante y de mano de sus potenciales clientes, para el lanzamiento de alguna campaña de marketing o la investigación de mercados de su respectivo sector.

\section{RESULTADOS Y DISCUSIÓN}

De las dieciséis preguntas realizadas con el instrumento de encuesta, se seleccionaron ocho; se consideraron aquellas que generan el mayor impacto como factor influyente en el diseño de campañas de marketing digital. De esta manera, se interpretaron las variables más representativas con la intención de generar conocimiento y contrastarlas con investigaciones nacionales e internacionales previas para ofrecer validez a los resultados. Es importante considerar la respuesta de las palabras claves usadas para buscar artículos del sector, para lo 
cual se determinó la moda de esta pregunta, encontrando que la palabra maquillaje se repitió 196 veces; adicionalmente, las palabras más usadas por los usuarios para buscar este tipo de productos fueron: make up, maquillaje, cosméticos, natural y belleza. Este es un dato importante a la hora de establecer estrategias de SEO y SEM, con el fin de lograr búsquedas orgánicas más eficientes en los buscadores de Google.

Para el cumplimiento del objetivo de la investigación, a continuación, se relacionan los resultados más representativos:

La Figura 2, indica que el $72 \%$ de los usuarios de las redes sociales de la marca Mirely Makeup, realizan sus compras entre las 2:00 pm y las 10:00 pm, considerando que la tendencia de consumo está orientada a ocho horas continuas de intensa publicidad en las plataformas tecnológicas, durante las cuales se realiza mucho contenido. Los Influencers eligen estos horarios para publicar sus videos, fotografías, tutoriales, talleres, cursos, etc. De igual forma, gran cantidad de la publicidad paga por empresas, genera tránsito en estos horarios, por lo tanto, son los predilectos para la generación de ventas efectivas.

Figura 2 - Horarios con mayor frecuencia de compra de artículos de maquillaje en internet

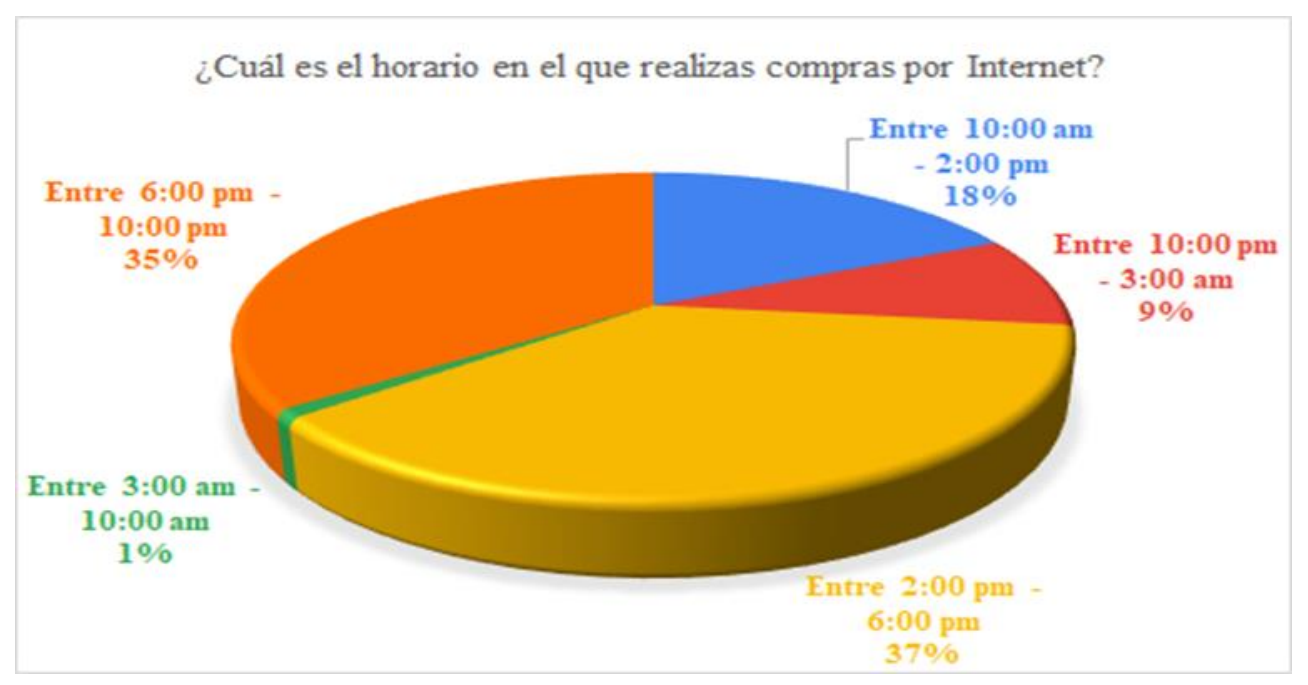

Elaborado por los autores

La figura 3, señala que el $85 \%$ de los usuarios utilizan canales digitales de compra y seguimiento de artículos del sector de maquillaje y belleza. Las páginas web de la marcas más posicionadas y reconocidas en el mercado se consolidan como plataformas de compra confiables para los usuarios, quienes han migrado hacia las compras digitales.

También se identifica el creciente aumento de compras directas en las redes sociales, las cuales se han afianzado como un canal de publicidad y venta masiva de variedad de productos, creando las llamadas tiendas virtuales en redes sociales, que en muchas ocasiones no requieren de sitios web de aterrizaje, concretando las ventas directamente a través de estos medios.

De esta forma, se ratifica el desplazamiento paulatino que han tenido los canales tradicionales de mercadeo hacia modos digitales, debido a la mayor rapidez en la difusión de información, disminución de costos, segmentación del mercado, alcance y efectividad (Figura $3)$. 
Figura 3 - Medios más usados para realizar compras

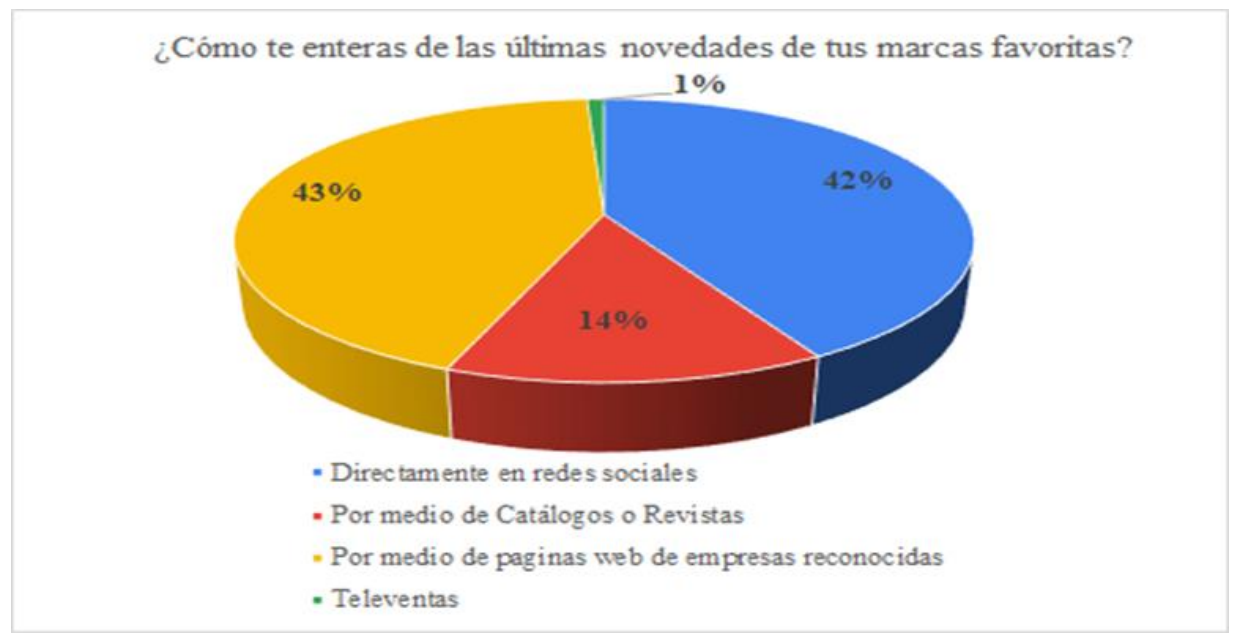

Elaborado por los autores

La Figura 4, indica que Instagram se consolida con el mayor porcentaje (82\%), como la red social más influyente en las decisiones de compra en tendencias de maquillaje. Esto obedece principalmente a las herramientas tecnológicas que posee esta red social, tales como historias, transmisiones en vivo, alimentación del feed con fotografías, videos, tutoriales, además de contar con los Influencers más famosos en los tiempos actuales, lo cual es algo muy atractivo por su carácter interactivo y dinámico que atrae público joven. Instagram es una plataforma enfocada al marketing; los hashtags permiten encontrar contenido rápidamente, compartir historias genera interacción entre los usuarios; los IGTV (Instagram TV) son una herramienta para cargar videos de mayor duración. La publicidad tiene estadísticas para medir en tiempo real los resultados de una determinada compaña. A mayor cantidad de seguidores de un usuario, Instagram va desplegando nuevas herramientas que generan mayores oportunidades de mercadeo.

Las grandes marcas tienen un perfil en esta red social, lo cual transmite una percepción a los seguidores de encontrar un portafolio de productos y servicios integral en esta red social. La interfaz de Instagram es sencilla de manejar y muy deductiva, al contar con alto contenido de material gráfico (video y fotografía) que cautiva el público objetivo.

Figura 4 - Redes sociales más influyentes en la decisión de compra de los usuarios digitales

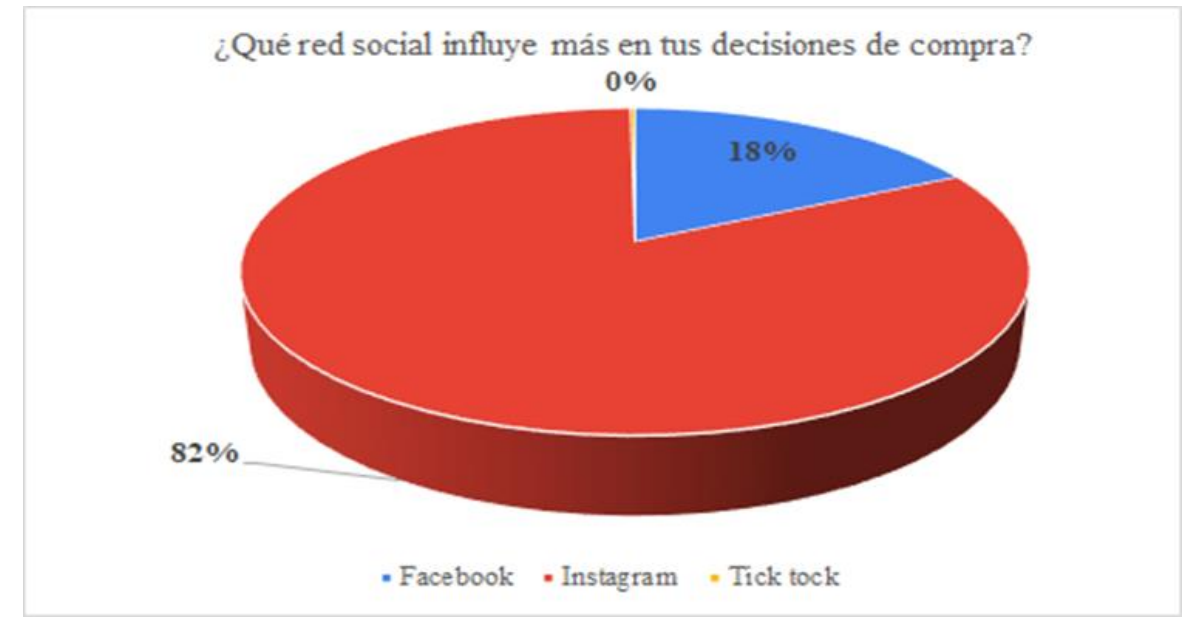

Elaborado por los autores 
La Figura 5, evidencia que el $33 \%$ de los encuestados siente confianza y es más perceptivo hacia las reseñas porque le interesa conocer el punto de vista y la experiencia de otros compradores para validar la confiabilidad de la marca; el $26 \%$ prefiere ser influenciado por tutoriales de maquillaje. En total, el $86 \%$ (reseñas, historias, tutoriales, videos) del contenido es video gráfico, siendo una herramienta más atractiva y destacada para la toma de decisiones de compra de los usuarios. En efecto, este tipo de contenido cuenta con un mayor desarrollo en la actualidad, debido a las aplicaciones que permiten crear y editar video de excelente calidad rápidamente a través de los celulares. Las personas se interesan más en un video que en una fotografía, puesto que ilustran y exponen de manera objetiva las cualidades de los productos y servicios, lo que permite concretar mayor número de ventas.

Figura 5 - Tipo de contenido más influyente en la toma de decisiones de compra en redes sociales

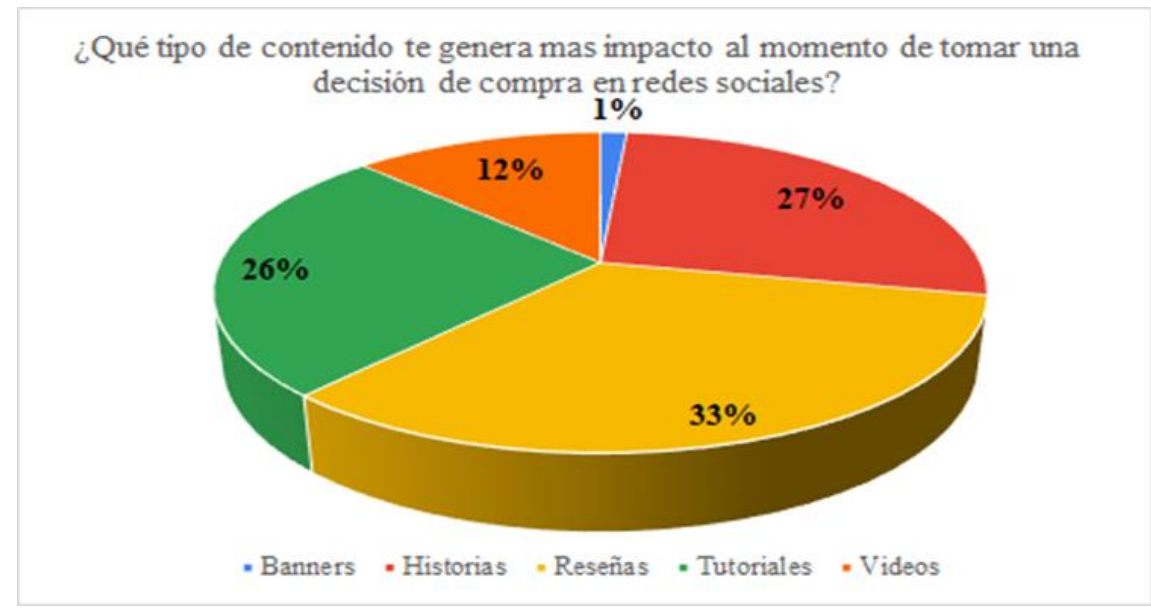

Elaborado por los autores

La Figura 6, muestra que un $61 \%$ de las personas realizan sus compras con normalidad entre semana. Probablemente este comportamiento obedece a que los usuarios en estos horarios están más dispuestos a comprar, ya que se encuentran laborando y motivados para realizar transacciones. Se identifica que en días laborares se estimula la compra de este tipo de productos por parte de los usuarios, ya que en sus espacios de descanso interactúan con las redes sociales y adquieren este tipo de productos. Por otra parte, cabe resaltar que contrario a la creencia, las personas ocupadas disminuyen la intención de compra los fines de semana puesto que se encuentran en actividades de esparcimiento familiar o actividades deportivas.

Figura 6 - Hábitos de compra de los usuarios del comercio electrónico

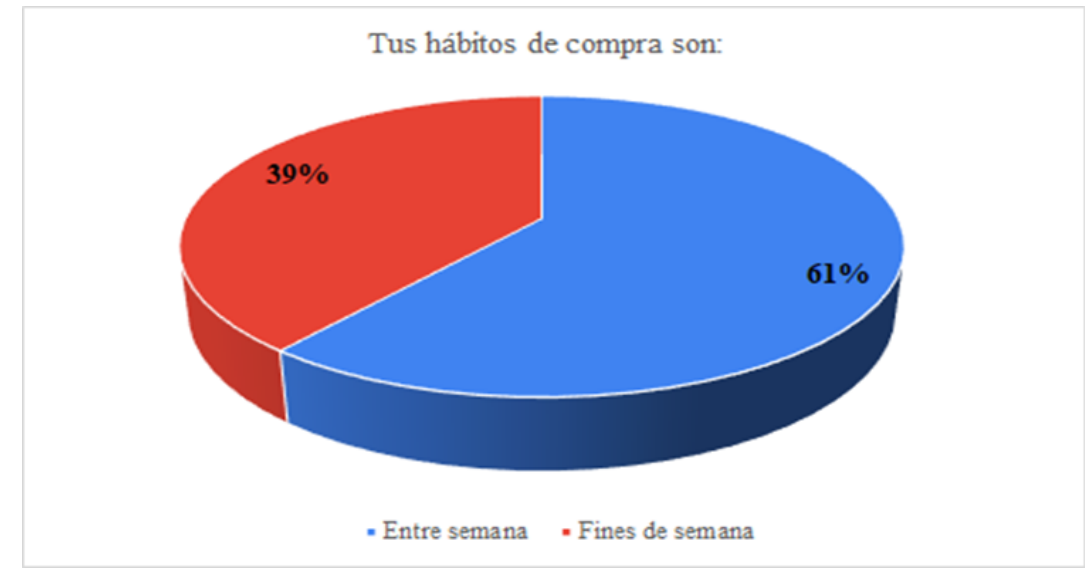

Elaborado por los autores 
La Figura 7, destaca que WhatsApp contó con un $67 \%$ de aceptación como medio de comunicación preferido por los usuarios de maquillaje en Colombia, convirtiéndose en el servicio de asesoría predilecto para concretar ventas con los usuarios interesados en recibir información a profundidad de productos y servicios. Es de resaltar la importancia que cobra esta red social en la actualidad, consolidándose como una canal de ventas por excelencia.

De acuerdo con las nuevas características de la plataforma, se generan soluciones más efectivas en la atención del público objetivo. La aplicación WhatsApp permite ser vinculada a otras redes sociales con mayor tráfico en la actualidad, mediante links, que conectan fácilmente desde Instagram y Facebook, lo cual a su vez genera confianza en las personas, quienes también buscan el contacto directo o atención de una persona a través de un mensaje o video llamada, buscando una relación más personal y humanizada.

Figura 7 - Preferencia de la herramienta digital WhatsApp, por excelencia en el servicio de venta y postventa en el comercio electrónico

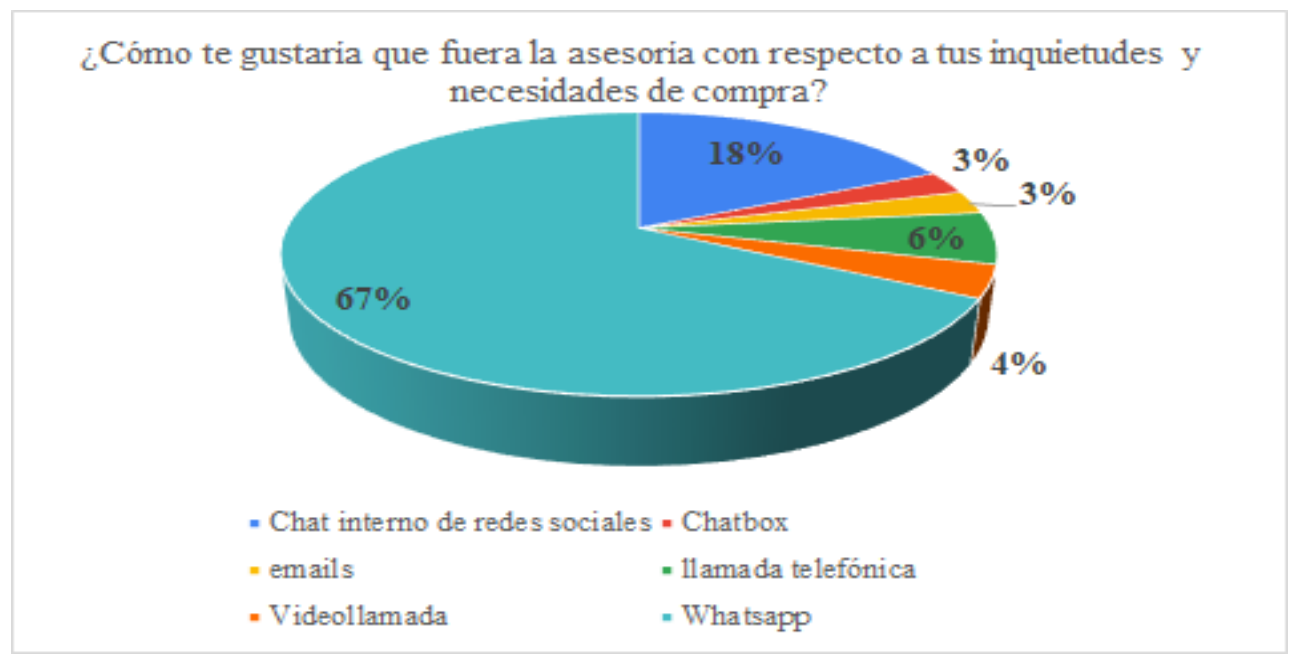

Elaborado por los autores

La Figura 8, evidencia el incremento de la apropiación del Email Marketing, como medio para orientar publicidad directa a usuarios que yacen en las bases de datos de las diferentes empresas. El $49 \%$ de las personas encuestadas dan click en los mensajes de correo electrónico que contienen publicidad directa con el objetivo de lograr la conversión de compra. Esto permite evidenciar el uso de esta herramienta de marketing digital, cada vez más generalizado como parte de las estrategias de marketing de las empresas de hoy. Esto obedece a que en la cotidianidad se autoriza el uso de información de contacto como el email, cuando se realizan compras en diferentes cadenas de Retail, o cuando se realizan suscripciones a páginas web o diferentes Marketplace. Por tanto, esta información se convierte en data de relevante importancia para las compañías, de tal manera que estas bases de datos adquieren un gran valor comercial. 
Figura 8 - Destaca el uso de la herramienta de Email marketing, como estrategia en campañas de marketing digital

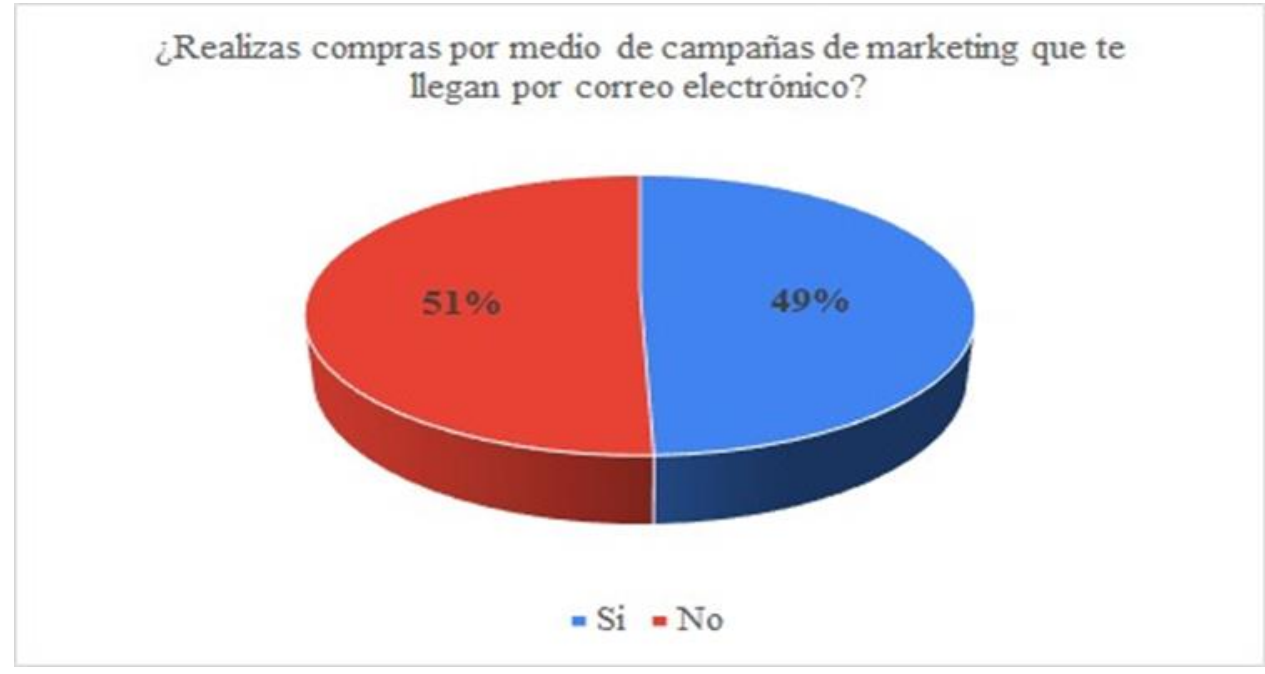

Elaborado por los autores

La Figura 9, destaca que, de acuerdo con el nivel de digitalización, el 37\% de los usuarios prefieren pagos tradicionales en efectivo. Esto es debido a que el nivel de transformación en pagos digitales se encuentra en un proceso de apropiación digital en Colombia; aún, no todas las personas cuentan con tarjetas de crédito, dinero electrónico y cuentas de ahorros. Sumado a esto, el nivel de confianza en los Marketplace no alcanza los niveles óptimos para que los usuarios se sientan totalmente cómodos con las transacciones electrónicas, es decir, algunas personas no se sienten seguras de digitar sus datos bancarios o de tarjetas de crédito para realizar transacciones on line, por lo que prefieren pagar sus compras en efectivo utilizando canales tradicionales como puntos Baloto, Efecty, SuRed o pagos contraentrega.

A pesar de esto, se observa un incremento representado en el $62 \%$ de consumidores que utilizan canales digitales de pago como botón PSE, Nequi, Daviplata, tarjetas de crédito y tarjetas débito. Esto obedece al esfuerzo que ha realizado la banca para integrar diversos medios de pago y acaparar los beneficios financieros que representa el comercio electrónico. Es así como nacieron las pasarelas de pago que se han integrado a los carritos de compra de los Marketplace, ofreciendo al usuario diversidad de opciones de pagos con el fin de concretar las ventas.

La tarjeta debito hace parte del pago por botón PSE, pues para usarla en los medios digitales se requiere usar necesariamente el botón PSE; por lo tanto, el medio de pago PSE se está usando en un 35\% y se evidencia su consolidación como una de las herramientas de pago más utilizadas como medio digital. Esto obedece a que el botón PSE es una herramienta práctica y fácil de usar que integra un gran portafolio de la banca tradicional, de tal forma que el usuario puede transmitir su dinero directamente desde de su cuenta de ahorros o corriente, dinero el cual es debitado de forma inmediata, logrando concretar la compra.

También, se refleja que el bajo uso en un $7 \%$ de las tarjetas de crédito es acorde al porcentaje de interés y cuota de manejo que utiliza este medio de pago, incrementando en el mediano plazo el costo de los productos. Sumado a esto, se comienzan a consolidar nuevas alternativas de banca virtual que no generan costos adicionales para el usuario, tales como 
Daviplata, Nequi, Bancolombia ahorro a la mano, tarjetas virtuales, las cuales son aplicaciones que permiten tener dinero digital disponible y realizar transacciones sin costo.

Figura 9 - Medios de pago de mayor aceptación en el E-commerce

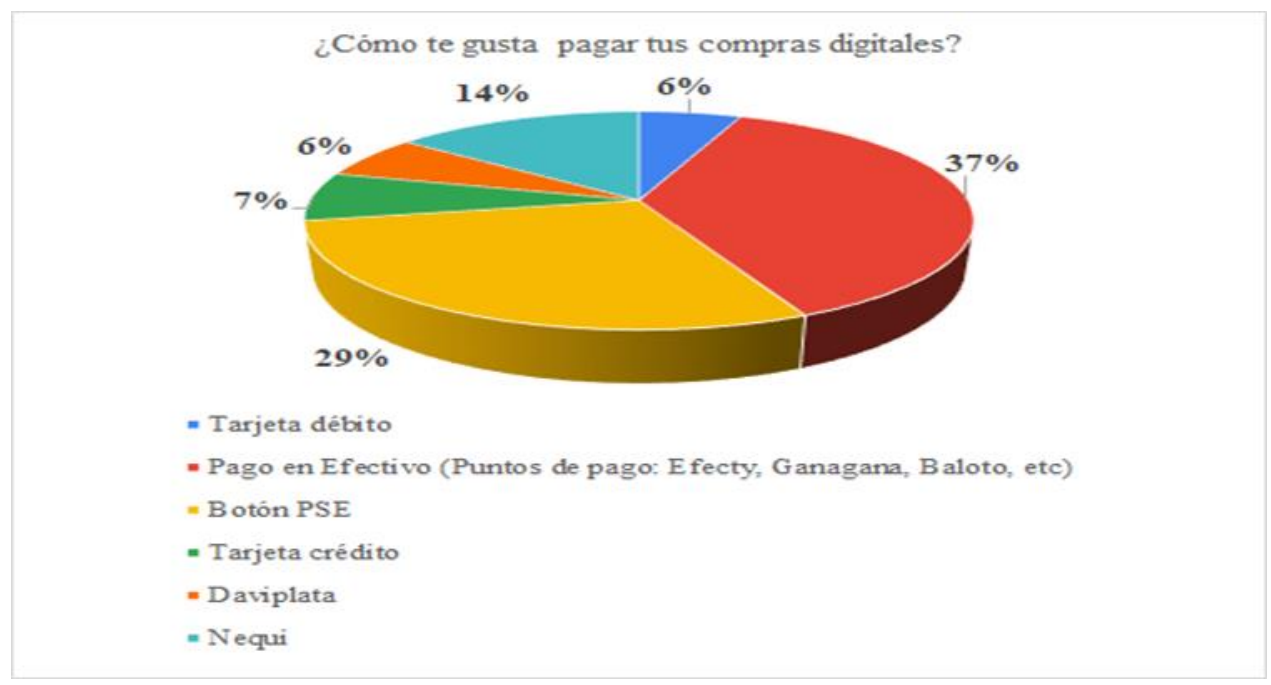

Elaborado por los autores

\subsection{DISCUSIÓN}

Esta investigación debe permitir una adecuada orientación al perfilamiento de los seguidores de las redes sociales de la marca de maquillaje Mirely makeup, que permitan a las empresas ahorrar costos de promoción, mediante la correcta segmentación del mercado, escogencia de horarios y fechas adecuadas de publicación, manejando los canales de pago más solicitados por los clientes, llegando puntualmente al segmento que se requiere e impactando en sus motivaciones y expectativas.

Después de analizar los resultados, se evidencia la preferencia a las compras de maquillaje por medios virtuales. Las páginas web de empresas reconocidas se han posicionado como la primera opción; sin embargo, se ha evidenciado un aumento en el volumen de compra a través de redes sociales. Debido a la globalización y conectividad, se ha desplazado a otros medios de compra incluyendo a las ventas por catálogo. Se resalta la omnicanalidad en el consumo y la búsqueda con intención de compra desde el celular, reduciendo el número de ventas por otros canales e incluso las compras offline, ya que los consumidores ven las transacciones desde un canal móvil como un canal de compra práctico y complementario. Lo anterior, es coherente con lo planteado por La Republica (2019), quien asevera que los colombianos cada día apuestan mucho más a los canales digitales para suplir sus necesidades.

Teniendo en cuenta lo precedente, las compras virtuales de maquillaje a través de Instagram marcan la tendencia, porque este canal de comunicación es más práctico, genera más confianza en el cliente y tiene disponibilidad de variedad de artículos al alcance de la mano. Este resultado está de acuerdo con Martínez-Rolán et al. (2019), quien argumenta que los usuarios pueden interactuar con el contenido publicado en Instagram desde cualquier dispositivo, lo que provoca que la opción de compra esté siempre disponible, se pueda acceder a cualquier producto y se genere comodidad, comunidad de marca y confianza. La red 
social Instagram se consolida en Colombia como una plataforma fundamental dentro de la estrategia de social media de las marcas, para lanzar nuevos productos, promociones, obtener visibilidad, construir imagen de marca, encontrar nuevas audiencias, y generar compromiso con los usuarios de las marcas (engagement).

De esta forma, diseñar un plan de marketing de contenido no es tan complejo, lo importante es conocer a los consumidores a profundidad y adaptar el plan de contenidos a las necesidades de cada marca; el objetivo final es atraer a los usuarios "voluntariamente" a los contenidos y luego mediante varias técnicas sugerirle realizar determinada acción (Barger et al. 2016). En relación con esto, los resultados de la presente investigación arrojan que el contenido visual, tutoriales y reseñas es parte fundamental y generan confianza en el posible comprador. De acuerdo con los estudios de Rodríguez \& Vásquez (2018), los videos se consolidan como una herramienta efectiva para marcas de belleza de lujo como CHANEL, dentro de su estrategia de Social Media.

De esta forma, la web visual es una tendencia que silenciosamente se está imponiendo en el contenido publicado en las redes sociales y estas a su vez han tenido que adaptarse para brindar más protagonismo al componente visual, el cual se ve reflejado en imágenes más grandes, mejor calidad de video, historias y transmisiones en vivo (González, 2014). Se pronostica que para el 2021 una persona promedio pasará 100 minutos cada día viendo videos en línea, en comparación con los 84 minutos en 2019. Se evidenció un crecimiento del $32 \%$ anual entre 2013 y 2018 en la visualización de videos, previendo que el video en línea y su gasto en publicidad aumentará lo equivalente a un tercio de todo el mercado de la televisión en 2021 (MarketingNews, 2019).

Por lo anterior, es importante resaltar que los días más frecuentes de compra online son entre semana, siendo de lunes a viernes donde más se registra actividad y búsquedas relacionadas a compra. Estos resultados concuerdan con Interactive Advertising Bureau (IAB) Spain (2019), estudio que resalta que esto es debido a que la actividad virtual de compra se registra desde mediados de la tarde hasta mediados de la noche, motivado por descansos y uso del dispositivo móvil en momentos de ocio o descansos entre actividades laborares, tiempo que los consumidores aprovechan para revisar plataformas y así mismo realizar pedidos de productos y promociones actuales que rondan en las redes sociales.

Por otra parte, la tendencia de compra nos indica que hay ocho horas de constante vigilancia y preferencia de compra, las cuales están comprendidas entre las 10:00 am a 2:00 pm y entre 2:00 pm a 10:00 pm, de productos de maquillaje a través de las redes sociales; esto representa una oportunidad e información clave para realizar publicaciones. Así lo confirman la investigación de Cabrerizo (2014), quien señala que la búsqueda de información es un proceso particular dentro de la compra, es decir, los usuarios emplean gran parte de su tiempo en encontrar productos que cautiven mediante un material audiovisual atractivo, en particular plataformas digitales que permitan establecer una conexión eficiente que brinde confianza y facilite la interacción entre empresas y consumidores. Efectivamente, la curva de búsqueda comienza un incremento lineal a partir de las 10:00 am hasta alcanzar su pico aproximado entre las 10:00 pm y 12:00 am, para comenzar su descenso en la madrugada; este comportamiento también lo registra el IAB Spain (2019), donde se evidencia que la conexión con los diferentes dispositivos (móviles, ordenadores y tablets) tiene un comportamiento lineal, comenzando la conectividad en las primeras horas de la mañana, manteniendo un crecimiento lineal durante el día, con un fuerte incremento a partir de las 16:00 horas hasta llegar a las 00:00 horas.

WhatsApp contó con un $67 \%$ de aceptación como medio de comunicación preferido de los usuarios de maquillaje en Colombia, convirtiéndose en un servicio de asesoría 
predilecto por los clientes, debido a características como la practicidad en la comunicación y la inmediatez. Esta plataforma integra servicios de creación de grupos, envío de mensajes de difusión, de voz, fotografías y video llamada. Esto concuerda con los estudios de Márquez et al. (2020), quien afirma que WhatsApp, más allá de ser una herramienta se ha convertido en la red social por excelencia, incursionando en el mercado europeo y latinoamericano en un 90 $\%$, con prospectivas de crecimiento para el 2020 de más de 1500 millones de usuarios. Su carácter no intrusivo, y su fácil disponibilidad multiplataforma (Android, IOS, BlackBerry, Windows phone) lo convierten en una herramienta de negocios por excelencia.

El email marketing mostro un $49 \%$ de efectividad como parte de la estrategia en campañas de E-commerce. Este resultado concuerda con las investigaciones de Valencia (2019), quien ratifica que el uso sencillo, económico y masivo del mailling, es una de las estrategias más fuertes de campaña que se realizan, demostrando gran efectividad y posicionamiento en la mente del consumidor. En este sentido Tarasova et al. (2020), recomienda que el email marketing debe describir y ser explícito en las metas que desea alcanzar en el cuerpo del correo y ser específico en las acciones necesarias para alcanzar esos objetivos. Adicionalmente, debe estar soportada en una estrategia consolidada de SEO marketing que oriente las búsquedas hacia el correo y una estrategia de redes sociales que contantemente reporte tráfico de usuarios.

De acuerdo con Dakouan et al. (2019), el marketing por correo electrónico es un tipo de marketing basado en el concepto de marketing de permiso. Consiste en el envío masivo de boletines, artículos, promociones y ofertas a través del email, haciendo parte de los elementos del inbound marketing que consiste en utilizar técnicas de marketing que intentan captar el interés de los clientes, compartiendo contenido creativo y útil que motive a diferentes acciones según los objetivos y planes de las compañías (Godin, 1999). Concluyendo y conforme las afirmaciones de Streltsova et al. (2019), el email marketing debe contextualizar el usuario proveniente de las redes sociales, consolidando y facilitando el proceso de selección de bienes y servicios, así como la fidelización del marketplace, optimizando el proceso de recompra.

El $62 \%$ de los usuarios prefieren utilizar canales electrónicos para pagar sus compras en las tiendas virtuales. Estos corolarios están de acuerdo con Guo (2014), quien asevera que esta tendencia promueve la viabilidad en el registro de las plataformas virtuales y la vinculación de datos sensibles para generar pagos digitales. Cabe resaltar que los hogares latinoamericanos cada vez más relacionan sus actividades diarias a la automatización de procesos operativos como pago de servicios y compras tradicionalmente realizadas en mercados físicos. De tal forma, que los sistemas de pago consolidan pagos comerciales aumentando la seguridad, reduciendo tiempos y costos de operación, incrementando la rentabilidad de las empresas, toda vez que hace más inmediato el dinero, generando liquidez y creando historia crediticia para ambas partes, a la vez que permite un mayor control contable sobre los ingresos de la compañía (Klapper, 2019).

El botón PSE es un sistema centralizado desarrollado por ACH Colombia, mediante el cual las entidades financieras virtualizan sus servicios. En los hallazgos obtenidos se evidenció una participación del $35 \%$ de pagos en línea por este canal, lo cual lo ratifica la investigación de Trujillo (2017), al evidenciar un incremento importante en el uso de esta plataforma en línea que obliga la apropiación del comercio electrónico, facilitando transacciones para consumidores y recaudos para empresas. Es necesario destacar que el posicionamiento de marca se debe a la sencilla interfaz y buen servicio que presta el operador, vinculando jóvenes y adultos a esta pasarela virtual. Este resultado es acorde con la dinámica adoptada en las condiciones de emergencia sanitaria por Covid19 en el país y las estrategias 
de reactivación económica, donde en los días sin Impuesto de Valor Agregado (IVA) en Colombia, se incrementó el pago por PSE en un $54 \%$ con 1.47 millones de transacciones, contribuyendo a la transformación digital.

De esta forma, las plataformas de pagos virtuales integran herramientas como ERP y $\mathrm{CRM}$, que permiten la integración de la gestión y seguimiento de clientes, y adicionalmente el monitoreo de indicadores contables y financieros que facilitan la toma de decisiones de la compañía, sin necesitar un alto nivel de infraestructura tecnológica, solamente con soporte de Internet y equipos que cumplan las funciones básicas de conectividad.

\section{CONSIDERACIONES FINALES}

Se puede concluir que los principales factores que determinan las características del consumidor se ven reflejados en una serie de elementos que adecuan las estrategias de marketing y aceleran el proceso de comercialización y posicionamiento de marca al ser aplicados en las campañas a través de la social network. Las tendencias de consumo son dinámicas y se adaptan a los cambios del mercado; en ese sentido, la identificación de canales efectivos de promoción y comercialización es esencial para acceder a nuevos segmentos de mercado y posicionar las marcas. Sumado a esto, la industria de belleza se caracteriza por la creatividad e innovación; por lo tanto, se debe propender por ser asertivos en el indicador de eficiencia de click y compra, por lo que es fundamental conocer horarios, tipos de promoción preferidas, redes sociales, contenido digital, entre otros aspectos, que permitan obtener herramientas para determinar hábitos de compra y adaptar las marcas a la necesidad requerida.

Conociendo las características más influyentes de los consumidores, es clave integrarlas a la estrategia, para de esta forma sacar provecho de la información y enfocar eficientemente las actividades que hacen parte de la formulación de campañas efectivas que logren el nivel de conversión deseado. Por lo tanto, se debe evaluar el entorno, el ecosistema y los stakeholder de la compañía para generar estrategias de fortalecimiento y posicionamiento. Con el diseño de las estrategias de marketing se espera que las metas sean alcanzables y los indicadores de cumplimiento se conviertan en insumo para la toma de decisiones futuras.

Por consiguiente, los diversos factores como SEO y SEM marketing, influencers, millennials, Instagram, Facebook, entre otros, desempeñan un papel importante en la ejecución de campañas exitosas de marketing digital, sin embargo, por si solas no determinan efectividad ni porcentaje de éxito, necesitan de un monitoreo y seguimiento constante que determine el avance de las metas propuestas y permita generar planes de mejora orientados a la consecución de los objetivos. Bajo esta perspectiva, se debe de considerar la estrategia de marketing como una iniciativa holística y sistémica que identifica las líneas de acción de la organización como un todo, integrando las áreas de la compañía en pro de la puesta en marcha y correcta ejecución del plan, ya que esto permitirá obtener retornos significativos que impactarán las demás áreas de la organización.

Por otra parte, cabe señalar que el tráfico de usuarios es considerable por plataformas como Facebook, Instagram, Twitter y YouTube, donde la información se visibiliza rápidamente y se obtienen resultados medibles a corto y mediano plazo. Estas redes manejan un alto contenido gráfico que vincula al consumidor y lo lleva a querer profundizar en los detalles y características de los productos, deseando tener un trato personal debido a la 
mentalidad y cultura de negocios tradicional. Por esta razón, WhatsApp desempeña un papel fundamental en la consecución de negocios, ya que el espacio para generar intención de compra está a cargo de redes sociales más gráficas, pero para ejecutar el negocio y donde se concentra el poder de negociación es en la red de mensajería, toda vez que se acerca más a un contexto más cercano al tradicional de negocios, permitiendo incrementar la confianza entre consumidores y empresas.

Finalmente, debido a la globalización, los consumidores digitales son más asequibles para las pymes, encontrando herramientas con bajo costo de inversión que les permiten acelerar sus compañías en el ámbito comercial. Identificar los factores que influyen en el diseño permitirá a las empresas diseñar su portafolio de soluciones acorde a las necesidades de los clientes, prevaleciendo el nicho de mercado objeto de campaña. Partiendo de una segmentación podrán implementar una estrategia que logre fidelizar consumidores y migren de compradores a clientes, representando embajadores de marca a bajo costo para las compañías; no obstante, los gerentes de las organizaciones deben tener claro que los factores identificados son una guía y la efectividad del plan dependerá del análisis del entorno interno y externo de la organización, lo cual cambiará la forma en que se deba estructurar la propuesta.

\section{REFERENCIAS}

ANDRADE, D. (2016). Estrategias de marketing digital en la promoción de Marca Ciudad. Revista Escuela de Administración de Negocios, (80), 59-72.

BARGER, V.; PELTIER, J.; SCHULTZ; D. (2016). Social Media and Consumer Engagement: A Review and Research Agenda. Journal of Research in Interactive Marketing, 10(4), 268-287.

CÁMARA COLOMBIANA DE COMERCIO ELECTRÓNICO (2020). Impacto del COVID-19 sobre el comercio electrónico en Colombia. Recuperado de https://www.ccce.org.co/noticias/covid-impacto-informe-comercio-electronico-colombia/

CABRERIZO, M. (2014). Procesos de Venta. Madrid: Editorial Editex S.A.

DAKOUAN, C.; BENABDELOUAHED; R.; ANABIR, H. (2019). Inbound marketing vs. outbound marketing: independent or complementary strategies. Expert Journal of Marketing, 7(1), 1-6.

DEISS, R.; HENNEBERRY, R. (2020). Digital marketing for dummies. John Wiley \& Sons

DESAI, A.; SHAH, J. (2019). Integrated Marketing Communication Then and Today-A Challenge or An Opportunity? Journal of Marketing Vistas, 9(1), 2-12.

DIEZ, F.; BLANCO, A.; PRADO, C. (2019). Research challenges in digital marketing: sustainability. Sustainability, 11(10), 28-39.

DODSON, I. (2016). The art of digital marketing: the definitive guide to creating strategic, targeted, and measurable online campaigns. John Wiley \& Sons.

FONSECA A. (2014). Marketing Digital en redes sociales: Lo imprescindible en Marketing Online. Madrid: ESIC. 
GODIN, S. (1999). Permission Marketing. New York: Simon \& Schuster.

GONZÁLEZ, M. A. (2014). Pinterest. La red social visual y creativa. Editorial UOC.

GUO, M. (2014). Relationship marketing in an online social media context: Newspapers versus brand websites comparison. Journal of Media Business Studies, 11(4), 1-26.

HERNÁNDEZ, R.; FERNÁNDEZ, C.; BAPTISTA, M. (2014). Metodología de la Investigación (6ta ed.). México: Mc Graw Hill.

INTERACTIVE ADVERTISING BUREAU (IAB) Spain, (2019). Interactive Advertising Bureau. Estudio anual de redes sociales.

KLAPPER, L. (2017). Ventajas del pago digital para los empresarios. IZA World of Labor.

LA REPUBLICA (2029). El auge del E-commerce en Colombia. Recuperado de https://www.larepublica.co/especiales/especial-ecommerce-julio-2019/las-transacciones-atraves-del-canal-digital-sumaron-512-billones-2891033

LÓPEZ, J. J et al. (2019). Digital marketing actions that achieve a better attraction and loyalty of users: An analytical study. Future Internet, 11(6), 1-16. doi:10.3390/fi11060130

MarketingNews (2019). La visualización de vídeo online alcanzará los 100 minutos al día por persona en 2021. Recuperado de https://www.marketingnews.es/marcas/ noticia/1154581054305/visualizacion-de-video-online-alcanzara-100-minutos-al-dia-persona2021.1.html

MÁRQUEZ, A.; CORROCHANO, M. C.; GONZÁLEZ, M. R. (2020). Redes Sociales (RR. SS.). Tres miradas a la participación de los jóvenes a través de las RR. SS. Participación cívica en espacios socioeducativos: Panorama iberoamericano en un mundo tecnológico, 2 , 91-122.

MARTÍNEZ-ROLÁN et al.. (2019). Instagram como red de promoción e hipermediación del turismo rural: el caso de Aldeias Históricas. Revista latina de comunicación social, (74), 1610-1632.

MÉNDEZ, M. (2013). El cibermarketing como estrategia para el fortalecimiento de la Mipyme en el municipio de Sincelejo (Colombia). Pensamiento \& Gestión, (35), 119-151.

MORA, P. et al. (2020). Las PYMES y su fortalecimiento con el uso de las TIC y el marketing digital. Polo del Conocimiento, 5(5), 420-438.

MOSCHINI, S. (2012). Claves del marketing digital. La nueva comunicación empresarial en el mundo 3.0. Barcelona: La Vanguardia Ediciones, S.L.

ORTEGA, M. (2020). Herramientas del marketing digital que permiten desarrollar presencia online, analizar la web, conocer a la audiencia y mejorar los resultados de búsqueda. Revista Perspectivas, (45), 33-60.

PERDIGÓN, R.; VILTRES, H.; MADRIGAL, I. R. (2018). Estrategias de comercio electrónico y marketing digital para pequeñas y medianas empresas. Revista Cubana de Ciencias Informáticas, 12(3), 192-208.

RASMUSSEN, L. (2018). Parasocial interaction in the digital age: An examination of relationship building and the effectiveness of YouTube celebrities. The Journal of Social Media in Society, 7(1), 280-294. 
RODRÍGUEZ, M.; VÁSQUEZ, I. (2020). EI vídeo tutorial como formato publicitario de los productos de belleza de las marcas de lujo. Estudio del caso Chanel en YouTube: uso, tipología, características y rendimiento. In Las redes sociales como herramienta de comunicación persuasiva. McGraw-Hill Interamericana de España.

SAINZ, J. (2018). El plan de marketing digital en la práctica. Esic editorial.

SANMIGUEL, P. (2020). Influencer Marketing: Conecta tu marca con tu público. LID Editorial.

SELMAN, H. (2017). Marketing digital. California, Estados Unidos: Editorial Ibukku.

SHIRLEY, H.; SASTOQUE, J. (2020). Marketing Digital como oportunidad de digitalización de las PYMES en Colombia en tiempo del Covid-19. Revista científica anfibios, 3(1), 39-46.

STATISTA (2020). Ranking de las 20 empresas líderes en el mundo en 2020, por valor de marca. Recuperado de https://es.statista.com/estadisticas/539088/ranking-de-las-20principales-marcas-en-el-mundo-por-valor-de-marca/

STRELTSOVA, E. et al. (2019). Information management system of industrial enterprise in conditions of digitalization. International Journal of Civil Engineering and Technology, 10(01), 559-575.

TARASOVA, E.; MATUSENKO, E; NAPLEKOVA Y. (2020). Modern marketing instruments for the company's market promotion. Journal of Research on Trade, Management and Economic Development, 13(1), 7-19.

TORRES, E. (2020). Digitalízate ¿Por dónde empezar?: Cómo transformar tu empresa para la era digital. Penguin Random House Grupo Editorial Perú

TOZAN, T; MANTEROLA, C. (2017). Técnicas de Muestreo sobre una Población a Estudio. International journal of morphology, 35(1), 227-232.

TRUJILlO, F. (2017). Análisis de las prácticas del e-commerce vinculadas al comercio internacional colombiano (Bachelor's thesis, Fundación Universidad de América).

VALENCIA, P. (2019). Valoraciones y estrategia para la implementación efectiva de un Plan de Marketing digital en las Pymes. Revista Científica Agro ecosistemas, 7(1), 52-57.

VALENCIA, A et al. (2014). Influencia del marketing digital en el proceso de decisión de compra. Revista Científica y Tecnológica UPSE, 2(1), 1-5.

ARRIETA, G. V. (2017). Marketing digital y su poder en la comunicación. Bogotá, Colombia: Repositorio Institucional Universidad Santo Tomás. 